\title{
1 Theorizing the Process of Boundary Enlargement
}

\begin{abstract}
This chapter demonstrates the theoretical underpinnings of the book. By discussing the framework of contentious politics, the chapter shows its advantages for analyzing complex realities and its ambition for a dynamic approach. The absence of an explanatory concept with regards to the changes over the movements' distinctive boundaries, nevertheless, allows us to introduce the process of boundary enlargement; a dynamic process which shows how the extension of boundaries allows social movement organizations to move beyond their delimited cognitive and structural perimeter, and adopt new practices and repertoires. Furthermore, the chapter underlines the importance of the meso-organizational level and the use of social movement scenes, and provides justifications for the book's focus on the factors of organizational structure, resources and identity.
\end{abstract}

Keywords: Contentious politics framework; Boundary enlargement process; Social movement organizations; Organizational structure; Resources; Collective identity

This research argues that the recent economic crisis has enabled the facilitation of a boundary enlargement process, which has affected social movement organizations (SMOs) both internally, in terms of their internal operation, but also externally, regarding their relationship with institutional actors. In order to demonstrate this, we base our explanatory framework on the literature of social movements, with particular focus on the Contentious Politics approach.

The study of processes and mechanisms in social movement studies dates back to 2001, when McAdam, Tarrow and Tilly introduced the Dynamics of Contention. Since the 1980 s, frame scholars criticized the static model of resource mobilization by suggesting a dynamic understanding of mobilization,

Malamidis, Haris, Social Movements and Solidarity Structures in Crisis-Ridden Greece. Amsterdam, Amsterdam University Press 2021 DOI: $10.5117 / 9789463722438 / \mathrm{CHO1}$ 
arguing that the 'decision to participate over time [is] thus subject to frequent reassessment and negotiation' (Snow et al., 1986, p. 467). Despite subsequent efforts to transform the static models of resource mobilization theory and the political process approach into more dynamic accounts (Tarrow, 1998), Dynamics of Contention was the first work to introduce a comprehensive theoretical framework.

Dynamics of Contention aimed to combine structural, relational and cultural approaches to politics. In order to achieve this, the authors suggest two steps. First, to approach social movements as one aspect of contentious politics, equal to strike waves, riots, civil wars, revolutions as well as nationalist mobilizations and processes of democratization. These events differ in many respects and therefore, they are often studied separately. Contentious Politics came to denounce that all of them 'have common causal properties instead of each constituting an entirely separate causal domain' (McAdam et al., 2009, p. 289). Moving forward, the second step claims that despite their differences, these forms of contention undergo the same mechanisms and processes that enable collective action to take place (McAdam et al., 2001, p. 4). According to this perspective, scholars should 'treat the causal properties as consisting of recurrent mechanisms and processes which in different combinations and sequences produce contrasting forms of collective claim making, from nonviolent to violent, from routine to extraordinary, from conservative to transformative' (McAdam et al., 2009, p. 289).

Adopting a relational perspective, McAdam et al (2001) argued that contentious episodes and events are processes which emerge from the combination of different sub-processes. Within the variety of contentious actions, the authors defined these sub-processes as mechanisms, namely 'a delimited class of events that alter relations among specified sets of elements in identical or closely similar ways over a variety of situations' (McAdam et al., 2001, p. 24). Mechanisms compound into processes, meaning 'regular combinations and sequences of mechanisms that produce similar (generally more complex and contingent) transformations of those elements. Distinct processes involve different sequences and combinations of mechanisms that interactively produce some outcome' (Ibid). The framework of Contentious Politics identifies combinations and sequences of mechanisms, which have different starting points, produce different outcomes and are developed within different contentious events like revolutions, wars, democratization processes and crises.

The process of boundary enlargement, like other social processes, does not exist in a vacuum. As demonstrated throughout the research, the process of boundary enlargement differs between actors; every actor had a different 
starting point and followed a unique path which will most probably result in different outcomes. Nonetheless, it was the need created by austerity that has enabled its full development and exposure. In order to provide a more comprehensive understanding of the process of boundary enlargement, we further specify its theoretical underpinnings.

\subsection{Why Boundary Enlargement?}

In 1996, Melucci argued that social movements are 'actions that imply conflict, solidarity and a breaching of the system limits' (Melucci, 1996, p. 30). The overcoming of systems' limits refers to the systems that 'ensures the production of a society's resources', the organizational system that 'makes decisions about the distribution of these resources', the political system that 'governs the exchange and deployment of the latter', and 'the system of reproduction in everyday life' (Ibid, p. 27). Within this approach, boundaries represent the limits of these systems and define the space in which movements negotiate with these four systems of power. In the volume of 2013 on The Future of Social Movement Research (Stekelenburg et al., 2013), leading scholars attempted to frame the current state-of-the-art in social movement studies. Among other issues, the contributors raised attention to the fluidity of identities as well as to actual and symbolic boundaries as central social factors shaping the development of collective action. Despite having almost 20 years separating them, both accounts underline the importance of limits and boundaries in collective action. Prompted by these, our research elevates the study of boundaries to the central issue at stake.

On the subject of the dynamic character of identities, Diani notes that boundaries rarely distinguish movements from their environments. On the contrary, 'we have boundaries that are often permeable, more or less dense areas of mutual recognition, and possibly chains of reaction' (Diani, 2013, p. 154). The definition of boundaries 'mirror processes of identity building, establishing connections across time and space, for example, within different phases of personal biographies, between generations, or between events occurring simultaneously in different locations' (Diani and Mische, 2015, p. 312). This approach adopts a relational perspective on boundaries, which enables their enlargement in practical and cognitive terms. Literature on social movements strongly suggests that the definition of boundaries be a crucial factor for the development of collective identity (Taylor, 2013). This deals mostly with groups and associations and their effort to create, sustain or reinforce their particular identities (Diani and 
Mische, 2015, p. 312). The definition of boundaries has implications not only for groups' identities, but also for their internal organization and operation. Here, boundaries play an important role in addressing conflicts as well as loyalties (Diani, 2015, p. 15). Shifting the focus from single organizations, groups or associations, to networks, boundaries may prevent or enhance the diffusion and exchange of practices and knowledge (Diani and Mische, 2015, p. 312). These may include the 'circulation of symbols, the expression of emotions, or the sharing of militancy and friendship' (Ibid). The role that boundaries undertake in enhancing the interaction between individuals, groups and networks, may also apply to broader schemata, such as social movements or even social fields. According to Diani, the definition of boundaries is rather essential for the connection of activists and social movements. The lack of formal membership criteria makes it difficult for activists to identify with certain movements, especially when the formers' lifestyles, values, beliefs and actions are not strong enough as determinants (Diani, 2013, pp. 152-153). Rather, the author notes that 'individuals may be associated with a movement to the extent that they recognize each other, and are recognized by other actors, as a part of that particular movement' (Diani and Mische, 2015, p. 312). In this sense, the definition of boundaries deals with the 'criteria that assign social actors to different groups and categories' (Ibid). Although Diani supports that there is balance in the definition of boundaries between organizational and movement level, at the same time the definition of boundaries refers to social action in broad terms, and affects our understanding of political systems, processes and dynamics (Diani, 2015, p. 16).

Social movement literature pays particular attention to the boundary definition. Due to this, the Contentious Politics approach has often stressed issues related to boundaries. The boundary definition discussed earlier transforms into a mechanism under the label boundary formation. This indicates the establishment of a rough separation of two political actors (Alimi et al., 2015, p. 287). Framed as a mechanism that can 'expand the range or extend the life of contentious episodes' boundary activation is a term used by Tilly and Tarrow for the 'creation of a new boundary or the crystallization of an existing one between challenging groups and their targets' (Tilly and Tarrow, 2015, p. 36). Boundary activation is also explained as the 'increase in the salience of "us-them" distinction separating two political actors' (Alimi et al., 2015, p. 287). Respectively, boundary control is a term used in order to describe the process of protecting the established boundary from outsiders (Ibid). Recent research work on boundaries introduced the concept of boundary-spanning (Wang et al., 2018). Boundary-spanning 
refers to the establishment of movement alliances as well as points out how movements conceive their goals and resources. The concept tries to bridge different insight to inter-movement and inter-organizational interactions, in order to study the transformative effects of boundary-spanning both in relation to social movements and with regard to social change at large. These terms are inter-related, as they all attempt to describe a process that affects specific boundaries. Tilly and Tarrow argue that boundaries are formed after complex possesses which 'commonly take shape outside contentious politics' (2015, p. 106). Once boundaries have been formed, 'political actors regularly use them as part of contentious politics' (Ibid), setting in motion the mechanisms of boundary activation and de-activation. As the two authors suggest elsewhere, although boundaries are transformed and new boundaries emerge, contentious politics neither create nor activate new boundaries; rather, they activate or de-activate the existing ones (Tilly and Tarrow, 2006, p. 61).

Contentious Politics scholars approach movements and their organizations as dynamic entities which change over time. Boundary formation is a term used in Contentious Politics to describe the process of establishing a new boundary. Similarly, identity shift, which denotes the 'formation of new identities within challenging groups' (Tilly and Tarrow, 2015, p. 37), constitutes a recurrent mechanism in many contentious episodes and often helps the explanatory framework of boundary transformation. However, there seems to be a profound absence of a term able to define the stretching of the practical and conceptual boundaries for SMOs. For this reason, we introduce the term "boundary enlargement". As mentioned earlier, by boundary enlargement, we refer to a process where previously defined boundaries are extended, enabling social movement organizations to move beyond their delimited cognitive and structural perimeter and adopt practices and repertoires that, up to that point, had been applied by distant and often antagonistic actors.

Among other processes, social movement studies, preoccupied with both movement outcomes and movement transformation, have often treated the processes of institutionalization and radicalization as the two extremes in terms of SMOs' development. The notion of institutionalization refers to 'the process of inclusion in the terrain of formal politics of some of its ideas (i.e., movement concerns come to be recognized as legitimate within mainstream politics and/or among the general public), personnel (i.e., activists gain positions within political parties, committees, and/or the civil service), or whole movement strands (i.e., sections of the movement establish political parties)' (Bosi, 2016, pp. 338-339). Respectively, the process of radicalization 
is defined as the 'process through which a social movement organization (SMO) shifts from predominantly nonviolent tactics of contention to tactics that include violent means, as well as the subsequent process of contention maintaining and possibly intensifying the newly introduced violence' (Alimi et al., 2015, p. 11). The reason why we provide these definitions lies in our effort to demonstrate that both indicate a paradigmatic shift that led SMOs either to the institutional side of practicing politics or to an outlawed one. ${ }^{1} \mathrm{On}$ the contrary, the process of boundary enlargement indicates that, although SMOs undergo a transformative trajectory that primarily affects their repertoires, but simultaneously influences their identities, organizational structure, resources as well as other aspects of their operation, they still remain active on the terrain of social movements.

The overlap between the rise of social movement research and the proliferation of organizational studies in topics that are not strictly business-oriented, has concentrated the interest of researchers in exchanging frameworks and views regarding the study of SMOs (Davis et al., 2005). Prompted by this effort, what seems particularly attractive is the transformative process of servicitization. This term has been coined within the field of business studies, to describe the gradual tendency of businesses to transform their area of interest from "goods or services" to "goods, services, support, knowledge and self-service" during the 1980 (Vandermerwe and Rada, 1988). Although serviticization is a process that partially reflects the incorporation of services that used to be, until now, provided by the welfare state and the market in SMOs' activities, its usage would not reflect the overall process that took place during the period of austerity, and thus it would be misleading for three reasons. Firstly, serviticization indicates that service-provision is the sole goal of these organizations. As such, this approach would lose sight of the element of social transformation, which is a key goal for the majority of the organizations studied. Secondly, the term implies that the provision of services is the only means by which businesses may respond to the transformation of the market. This would be a second misconception, since it would exclude other aspects of SMOs' repertoires that are not orientated to the provision of services. Additionally, it would imply that this orientation was solely imposed indirectly by welfare

1 It is worth noting here, that according to some scholars the movements' institutionalization does not necessarily diminish their antagonistic character (Dee, 2018). Although this might be true, it is rarely the case. Additionally, in those contexts, institutionalization refers to the partial adoption of formal characteristics and does not correspond to the movements' engagement with mainstream politics and political parties, as is the case with Bosi's (2016) definition. 
retrenchment, thus leaving out firstly the agency of SMOs to decide upon their own practices; secondly, the critical stance of some SMOs towards service-provision; and thirdly, the approach of pre-figurative politics that led some of these organizations to turn to actions that would reflect the envisioned society. Lastly, serviticization underlines a strategic behaviour of a specific set of actors, namely businesses, while boundary enlargement indicates that the SMOs' boundary expansion takes place in a field of conceptual and cognitive understanding that may touch upon the boundaries of other actors, such as movements' relationships with the state.

Before we proceed further, it is important to provide some clarifications regarding the definition of boundary enlargement. While reviewing the relevant literature on boundaries, we came across topics related to identities. Many authors (Diani, 2015; Staggenborg, 2013; Tilly and Tarrow, 2015) have stressed that boundary definition is quite essential for the definition of identities. Although this is true, boundaries are also important for resources and organizational aspects as discussed throughout this study. Nevertheless, the transformative procedure, where stable, solid and well-defined boundaries tend to change shape, features heavily in late and post-modern accounts.

Marshall Berman (1983) in the 1980 os provided an account on how modernity could be pictured as the way forward for further developing an inclusive understanding of the modern world. Some 30 years later, Zygmunt Bauman (2007) described how structured modernity becomes fluid within neoliberalism. Although the former account embraced the goods of an era that "development" meant to be something good, the latter one describes emphatically how the current social structures and norms become liquid and should not be expected to return in their previous stable condition. On the same vein, Melucci (2002) underlines that former integrated subjectivities transform and mark a fluid sense of identity. Late and post-modern accounts argue about the complexity of the contemporary world and the dissolution of values, structures, norms and institutions that used to be taken for granted. Like every social process, the aforementioned changes are rather lengthy and are being developed through incremental steps over the time. Although our empirical analysis shows that the enlargement of boundaries started to take place prior to 2010, the recent economic crisis and the harsh austerity policies have affected great parts of the economic, political and social environment in Greece and accelerated this transformation.

Moving onwards, we argue that the tendency observed in the Greek social movement community towards the provision of informal welfare services does not reflect only the enlargement of boundaries in conceptual 
terms, as boundaries are usually analysed in relation to social identities; it is also associated with the practical aspects of social movement repertoires, organizational structure and resources. This enlargement tends to open the relatively close structures and defined operations of SMOs, while also revealing new insight to the movements' understanding of social change and their association with institutional actors. One may ask at this point whether there is a sequence of changes, or if these changes occur simultaneously. Although we cannot assume a qualitative primacy of one factor over another, we can, nevertheless, claim that there is a chronological sequence. As we suggest in our empirical analysis, changes in repertoires triggered respective developments in SMOs' focus, with the latter's organizational structures and resources somehow adapting to the environment, in order to better serve the operation of the "new" alternative repertoires. Researchers suggest that shifts in a movement's tactical boundaries can signal respective changes in its cultural identities (Wang et al., 2018, p. 182). In this respect, the enlargement of boundaries with regards to identities is a much slower process and the outcomes of this change are not easy to grasp comprehensively. This shift towards the alternative repertoires of action was welcomed by many activists and criticized by others. This "conflict" highlights the pre-figurative dimension of the new, "in-the-making" identity but also emphasizes that its antagonistic and contentious character is not based solely on a claim-based orientation.

However, the application of this process is also limited, as it primarily reflects changes in social movement repertoires of action, and second, it is contextualized in the environment of rampant austerity. This does not mean that similar changes in other settings cannot be studied through the lens of boundary enlargement. Rather, we raise attention that this process, which so far mirrors the ultimate stage of consecutive shocks and changes in the Greek reality, in other contexts might represent an intermediary stage. Similar to the punctuated equilibrium theory in public policymaking, which explains that sharp changes complement the gradual institutional adjustments (True et al., 2007), Fligstein and McAdam (2012) argue that balance is always achieved after periods of change. From a post-modern approach, we neglect that the future post-crisis setting will find the social movement community identical to the pre-crisis one, but we cannot be sure whether a new process will be a successor to the boundary enlargement. In addition to this, the fluid nature of boundaries favours their transformations, which are now directed towards enlargement, but in other cases and under different circumstances, it might lead to boundary compression, reduction or contraction. 
The incorporation of service-oriented repertoires by the social movement community in Greece has not always been appreciated. Critics point to the vulnerable nature of these repertoires that can easily lead to movements' cooptation, while others emphasize the shift of activists' attention away from their subversive mission. Although these views are thoroughly analyzed and debated in the following empirical chapters, they raise awareness regarding the term "enable", used to define the boundary enlargement process. Studies on collective action usually focus on mobilization, and thus concentrate their attention on protest events. As such, they quite often obtain a critical stance towards other forms of action, since these reduce resources from the movements' central goal. Though by no means do we underestimate the value of protest and public claim-making with regards to broader social transformation, at the same time, we do not reduce the movements' role to the street level. In particular, we perceive movements and their organizations as bearers of social change, which enhance politicization, create and transmit progressive symbols, values and beliefs also in the context of everyday life during periods of silence.

This issue reveals a broader problem on social movement studies, since definitions of social movements have been predominantly based on the explanatory context of each research. Diani (2015) understands movements as a form of coordination with intense resource and boundary exchanges; Della Porta and Diani (2006) underline the network structure of movements; Tarrow (1998) and Tilly (2004) focus on the movements' ability to sustain campaigns against specific claimants; while McCarthy and Zald (1977, pp. 1217-1218) accentuate the participants' shared beliefs and opinions. This research acknowledges that the aforementioned definitions are not opposed to, but rather complement one another. Thus, in order to define the characteristics that constitute a social movement, we follow an inclusionary approach as expressed by Snow. More precisely, social movements are

change-oriented in the sense that they seek or oppose change; [...] challengers to or defenders of existing institutional structures or systems of authority; [...] collective rather than individual enterprises; [...] act outside of existing institutional or organizational arrangements; [...] operate with some degree of organization; [...] and typically display some degree of temporal continuity (Snow, 2013, p. 1201).

\subsubsection{Enlarging the boundaries at the meso-level}

The process of boundary enlargement in this inquiry treats the shift of SMOs towards service-oriented repertoires of action as its central aspect. However, 
the provision of social services as part of social movements' repertoires is in no way new. Similar efforts, such as the organization of mutual-aid funds, can be traced throughout the history of the labour movement. In his systematic categorization of organizations that constitute a social movement, Kriesi highlighted that together with SMOs, supportive organizations, parties and interest groups, the movement's formal associations are the fourth type of organization that complements the picture. Kriesi defined those as 'self-help organizations, voluntary associations, or clubs created by movements themselves in order to cater to some daily needs of its members', which 'contribute to the mobilization of a movement's constituency, but they do so in an exclusively constituency - or client-oriented way' (Kriesi, 1996, pp. 152-153). These organizations may create commitment or consensus to mobilization, but contrary to the SMOs, 'they do not directly contribute to the "action mobilization" or the "activation of commitment" for a "political goal"' (Ibid). As the author elaborates, 'if the constituents and the beneficiaries of the organizations are identical, we may speak of self-help groups or clubs; if this is not the case, we deal with voluntary associations engaged in altruism' (Ibid: 365 -footnote in the original).

The incorporation of interest groups in the organizations that constitute a social movement is rather debatable since it stretches the definition of social movements. Non-governmental organizations (NGOs), political parties, interest groups and other official forms of political action might be sympathetic to a movement, while SMOs might also use official or institutional means to defend their agenda (Diani, 1992, pp.13-15). Nonetheless, the incorporation of the former organizations in the definition of the latter runs the risk of creating a vague, catch-all term which provides little analytical explanation. What interests us in Kriesi's account, nevertheless, is that the author's categorization establishes distinctive lines between SMOs, whose main goal is to mobilize people and movement associations which do not share the same intention

Rucht's categorization of the organizations affiliated to social movements is connected to Kriesi's analysis. Rucht distinguishes six types of collectivities: basic action groups with local focus consisting of around 20 members, such as citizen initiatives; movement organizations and umbrella organizations, whose members and area of interest may vary from dozens to thousands and local to national respectively, but they are subject to formal rules, memberships, leadership; campaign networks and enduring networks, with the former pointing to ad hoc collaborations around a single issue with limited time and the latter referring to permanent collaborations which raise various issues and connect usually on an ideological basis; material 
and immaterial service structures, such as educational centres, bookstores, training clubs, fora or indymedia; social retails, such as self-organized cafes; and supportive social milieus, like specific jargons, lifestyles and consumer choices (Rucht, 2013, pp. 171-173). According to Rucht, 'the first four are ultimately geared toward action mobilization, whereas the last two provide a ground for consensus mobilization' (2013, p. 173).

These accounts touch upon two very important issues. The first one concerns the definition of SMOs and the second deals with the issue of actual mobilization. Starting from the former, both Kriesi and Rucht seem to conceive SMOs as the respective equivalent of what organizational studies consider formal organizations in social movements, which imply rigid boundaries that can distinguish one organization from another. Formal organizations have specific decision-making models, membership, they follow a hierarchical structure, impose specific rules, and have the right to monitor as well as to implement positive or negative sanctions (Ahrne and Brunsson, 2011). On the same basis, Diani (2015, p. xvii) argues that 'many organizations are embedded in structural patterns that somehow reflect social movement mechanisms without matching at all the stereotypical traits of "social movement organizations"'. Nevertheless, by introducing the concept of 'hybrid organization', an organizational type which consists of only specific features out of the ones that characterize the formal organizations, organization studies note the dynamic character that organizations have (Ahrne and Brunsson, 2011). Taking into account the informal character that social movements have, Kriesi and Rucht's approaches tend towards structuralism and rarely correspond to the horizontal and self-organized collectivities found in the field. The process of boundary enlargement, however, serves to demonstrate how these fixed boundaries dissolve into, on the one hand, traditional SMOs providing social services, and, on the other hand, Kriesi's movement associations or Rucht's social retails contributing to action mobilization.

The second issue deals with the literature of mobilization and participation in collective action. Grievances, discontent, material benefits, solidarity, purposive and selective incentives as well as structural factors, like history of prior activism or biographical availability, constitute few of the fruits of social movement research, which managed to reveal the reasons behind someone's decision to engage with collective action (Staggenborg, 2011, pp. 32-33). Walgrave (2013) notes that literature on mobilization can be roughly divided in two streams: the structural and the cultural. The former focuses its attention on SMOs' efforts to reach out to their sympathizers and broader audiences by arguing that the individuals' structural position 
is a strong proxy for participating in a movement; while the latter supports that the individuals' participation is dependent on their agreement with the movements' goals (Walgrave, 2013, p. 206). According to Walgrave, debates exist also within the culturalist stream, where some scholars argue that individuals participate in SMOs once the latter manage to reach consensus mobilization (Klandermans and Oegema, 1987, pp. 519-520) or align their frames with the needs of potential participants (Snow et al., 1986); while others claim the individuals' agency 'as active attributors of meaning constructing their own ideas and searching for opportunities to put these ideas into practice' (Walgrave, 2013, p. 206).

Drawing mostly from frame theories (Snow et al., 1986), Jasper and Poulsen (1995) also pay attention to specific instances and features that lead towards mobilization and the recruitment of activists. With regards to the animal rights and anti-nuclear movements, they argue that the recruitment of movements' sympathizers takes place 'through proximity, affective bonds' (Jasper and Poulsen, 1995, p. 508), but when it comes to the recruitment of strangers, cultural meanings and moral shocks seem more important. Moral shocks can be the outcome of SMOs' strategies. However, these can also be triggered during suddenly imposed grievances or in the individuals' everyday experiences and develop 'a sense of outrage in people that they become inclined toward political action' (Ibid, p. 498). Although it is still debatable whether networks are the pre-condition for or the outcome of the development of social movements and activists' recruitment (Diani, 2015), Jasper and Poulsen claim that 'cultural meanings and moral shocks may be especially important as a substitute when social networks are missing' (Jasper and Poulsen, 1995, p. 509).

Perceived as a suddenly (but continuously) imposed grievance, the economic crisis has managed to mobilize a number of people without previous experience in collective action. However, this was not limited at the street level; it also continued with the recruitment of many non-activists in the employment of the relatively silent alternative repertoires. Our research shows that the experience of a moral shock was quite decisive for many individuals to join social clinics or food-related initiatives, and it also encouraged others to establish cooperatives.

Although we proceed to provide additional information in the following parts of the book, it is important to make some clarifications regarding these two aspects and the three respective social movement scenes. The food social movement scene consists of grassroots organizations, whose origins depart from social movements, and employ an anti-austerity stance. Thus, according to Kriesi's categorization they could be framed as movement 
associations (Kriesi, 1996, p. 153). Following a similar path, social clinics do not constitute political organizations in the strict sense of having one goal and mobilizing people towards it. Rather, they employ their services and offer indirect support to the struggle against austerity in the healthcare sector. Therefore, the clinics could also be framed as movement associations (Ibid). Finally, the vast majority of cooperatives are closely linked to the anti-austerity mobilizations and enhance commitment to self-management, but they do not directly contribute to the mobilization of their constituents. Thus, the actors operating in the social movement scene of labour fit the definition of supportive organizations (Ibid, p. 152).

Although at first glance this categorization appears to correspond with Social Movement Industries (SMIs) (McCarthy and Zald, 1977), it does so only partially. ${ }^{2}$ On a number of occasions, the coercion against markets without middlemen by state authorities during the first years of their operation resulted in the mobilization of participants. Additionally, the plethora of organizations and traditional SMOs, which employ collective and social kitchens and initiatives for the collection and distribution of food parcels as additional aspects of their repertoires, are factors which prevent us from labelling these organizations as movement associations. Similarly, in the social movement scene of health, SMIs seem to occasionally participate in mobilization. Many clinics have launched initiatives which aim to inform their beneficiaries about their role, goals and operation, and distinguish themselves from institutional healthcare providers. As a matter of fact, the clinics have both participated in and organized protests against austerity and other political issues, successfully mobilizing their beneficiaries on a number of occasions. The establishment of clinics as an additional form in the repertoire of traditional SMOs, like the workers' club of Nea Smirni, are factors that contribute to this argument. Lastly, the examples of the occupied factory of Vio.Me which directly mobilizes its constituents, the $\mathrm{K}-136^{3}$ initiative against Thessaloniki's water privatization which led to the activists' collaboration with municipal authorities, as well as the incorporation of cooperative structures in the repertoires of traditional SMOs, blurs the picture in the social movement scene of labour.

2 Social movement industries (SMIs) refer to 'all SMOs that have as their goal the attainment of the broadest preferences of a social movement", while by social movement sector (SMS) we refer to "all SMIs in a society, no matter to which SM they are attached' (McCarthy and Zald, 1977, p. 1219). Although we do not adopt the definition of SMOs as it was introduced by McCarthy and Zald, we acknowledge the aforementioned typology in order to schematically represent the organizations which employ the service-oriented repertoires of action.

$3 \mathrm{~K}-136$ stands for Movement 136. 
The aforementioned contradictions serve to demonstrate that the boundaries among the SMOs, supportive organizations and movement associations, as these had been defined by social movement scholars (Kriesi, 1996), do not apply anymore. Of course, we do not opt to disregard them completely. Rather, we want to underline that the current economic crisis has facilitated a process of boundary enlargement, through which these fixed boundaries became interconnected. We should note here that the degree and intensity of interconnectedness varies between different actors and social movement scenes. For example, it might be the case that the cooperative means employed by SMOs pre-figure the ideal of self-management, but a social cooperative still remains an enterprise. Additionally, the markets without middlemen, which have been organized by neighbourhood assemblies, might proclaim people's empowerment but often lack a specific ideological or political orientation. Therefore, we do not argue for the complete dissolution of the respective boundaries of each organization. Instead, we point out their enlargement. In other words, the enlargement of boundaries reveals a process where tasks previously attributed to specific organizations are being challenged, re-defined, mixed and applied by both new and traditional SMOs. Now that we have delimited the theoretical approach of this research, we turn our attention to the construction process of boundary enlargement.

\subsection{Dismantling the process of boundary enlargement}

So far, we argued that the process of enlargement crosses the boundaries of SMOs, supportive organizations and movement association. The question that directly arises is how the process of boundary enlargement takes place. Tilly and Tarrow claim that 'distinct processes involve different sequences and combinations of mechanisms that interactively produce some outcome' (2015, p. 29). On the contrary, Alimi et al. (2015) take a slightly divergent approach by perceiving processes as defined by the attainment of a specific outcome. In other words, although both accounts come from the Contentious Politics approach and engage in dialogue together, they have different starting points. More precisely, Tilly and Tarrow argue that the identification of specific mechanisms leads to specific processes, while Alimi et al. support that it is the process that directs the researcher in identifying specific mechanisms. If $\mathrm{X}$ stands for a mechanism and $\mathrm{Y}$ for the process, the former account supports that the combination of specific Xs lead to $Y$, while the latter argues that it is the $Y$ that defines which Xs took place. In 
addition to this differentiation, Alimi et al. argue that a process should not be treated as an exhaustive account of mechanisms which provide causal inferences, since other mechanisms might also take place. This updated account also introduces the idea of sub-mechanisms as constitutive elements of mechanisms, which are defined as such according to the specific context (Ibid, p. 30).

This research adopts the epistemological account proposed by Alimi et al. (2015). More precisely, it does so by conceiving the enlargement of SMOs' boundaries as a process that took place during the period of crisis, and, therefore, it goes backwards in order to identify the mechanisms and sub-mechanisms that enable it to take place. In order to do this, this research focuses on the social movement scenes of food, health and labour.

\subsubsection{Social Movement Scenes}

As mentioned earlier, we intend to better explain the development of boundary enlargement process, through the activities of SMOs with regards to the scenes of food, health and labour. In the introductory part we provided the criteria used for the selection of the specific cases under study, while the next chapter on the background context presents the empirical evidence grounding this decision. Nevertheless, the decision to look at social movement scenes also includes some theoretical associations that need further clarification.

Our decision to refer to the scenes of social movement activity, such as food, health, and labour was based on the interaction of our empirical material with related studies on social movements. Recent academic scholarship in the area of social movements introduced important concepts that might help the conceptual construction of this research. Jasper and Duyvendak's Players and Arenas (2015) invites us to study the interactions among different actors preoccupied with a specific thematic. Thus, in our case, food, health and labour may constitute different arenas, with Jasper and Duyvendak's dynamic framework giving us the potential to study how the different actors in each arena interplay with each other. In this respect, the arena of food may consist of the institutional actors, such as the municipal authorities organizing soup kitchens and food donations, the national government imposing amendments on laws related to the application of open markets, a number of institutional social services as well as a few left-wing parties involved in the provision of food-related services; the business sector with the local groceries shops donating groceries to individuals in need, large food retailers co-organizing with institutional actors food donations, and 
brokers' associations raising pressure to the government in order to secure the former's role in the food retail industry; a set of NGOs involved in the provision of soup kitchens; and finally, the grassroots actors organizing bottom-up markets without middlemen, collective and social kitchens, and distribution of food parcels. A similar variety of actors can be observed in the health and labour arenas.

Although inspiring, the aforementioned analytical framework moves beyond the scope of this research. Our study takes into consideration the different actors that operate in the three respective arenas and explores their relationship with the grassroots collectives, but the focus of this research is the exploration of the enlargement of the movements' boundaries and not the development of the arenas per se. As we stated earlier, the sectors of food, health and labour play a rather instrumental role in this study; they are cases which serve to demonstrate how social movements in Greece, and SMOs in particular, experienced a transformative process which enabled them to engage with activities that were not in their direct area of concern. Therefore, we are less interested in analyzing the different actors that were at play, and much more in focusing in depth on social movements.

Another work that could be used as a point of reference for this study is Fligstein and McAdam's Theory of Fields (2012). The structure of our research has many points in common with what Fligstein and McAdam described as strategic action fields, and the reader will probably understand that our reference to the social movement scenes of food, health and labour might provide similar application with what field theory suggests. In Fligstein and McAdam's field theory, social movements would mirror one actor trying to challenge the social order of the field, as this is imposed by the incumbent actors, like the state and market promoting social welfare; and the internal governance units, like the organizations of the third sector trying to secure the status quo. In these terms, the process of boundary enlargement would be framed as an "invasion" (Fligstein and McAdam, 2012, pp. 99-100) due to the changes in the respective fields caused by austerity, showing an action of appropriating already populated social spaces.

Although tempting, we do not fully incorporate this approach for three important reasons. First, the focus of our research is not on the specific fields of food, health, and labour; rather, these are chosen in order to better explain the overall "invasion" of social movement actors in a wide variety of fields. Our reference to the social movement scenes aims to demonstrate the plurality of grassroots actors and approaches engaged in this "invasion", as well as their contradictions, that cannot be synthesized in one, single and solid actor. Second, our research is interested in exploring the changes 
in the social movement community by observing the meso-organizational level. In this respect, the field approach acquires an instrumental usage, which is not the case in Fligstein and McAdam's Theory of Fields. Lastly, our third concern lies in the overall perspective of field theory as described in Fligstein and McAdam's study. In particular, the authors' great emphasis on the reproduction of fields, and thus the status quo, as the final result of incumbent and challengers' actions, as well as their rendition of actors' moves on strategic choices, bears a deterministic connotation coupled with a rational approach concerning the actors' deeds. Although we do not disagree that some actions which followed this "invasion" were the outcomes of the movements' strategic planning, our analysis suggests that the moral shock that the Greek society experienced, social movements included, due to the vast austerity policies affecting everyday life, was the basic component of the boundary enlargement process and its related actions. This becomes particularly clear with regard to the scenes of health and food, as movements start to interact with state actors, deal with internal contradictions, and are confronted with new dilemmas that deviate from ideological purity. In this respect, the deterministic approach of field equilibrium after the shock that Fligstein and McAdam (2012, pp. 83-86) advocate for, tends to ignore the changes within the various social movement scenes and the broader social movement community.

Although theories on arenas and fields do not seem to conceptually correspond with the needs of this inquiry, social movement scenes seem to be the most adequate approach, which also allow to proceed to some within-case comparisons. According to Haunss and Leach (2007, p. 73), a scene is 'a network of people who identify as part of a group and share a certain belief system or set of convictions, that is also necessarily centreed around a certain location or set of locations where that group is known to congregate'. Earlier, we mentioned Diani's (2015) argument that individuals' membership in a movement depends on other participants' recognition as such. The same pretty much goes for the scenes, which are constructed based on mutual recognition. This leads to an essential element linked with the process of boundary enlargement, namely that 'scenes are selfconstituted dynamic entities whose internal and external boundaries are constantly in flux' (Haunss and Leach, 2007, p. 73). By studying the German autonomous movement, Hauns and Leach note that scenes are less culture-driven than sub-cultures, incorporate wider social characteristics than countercultures, and are 'less determined by cultural and economic capital' than milieus (Ibid). Additionally, in a later paper, the two authors note that scenes incorporate all the characteristics of pre-figurative free 
spaces, such as egalitarian relationships and an organizational model based on the participants' vision for the future society; while they rarely encompass features met in trans-movement and indigenous free spaces (Leach and Haunss, 2009, p. 256). In both papers, the two authors argue that scenes produce and sustain their own culture. In this respect, scenes do not always have a political orientation. When they do, it is not always driven by progressive political values (Ibid, p. 258-259). Nevertheless, when scenes mirror the central values of social movements and are tightly linked with them, we may speak of social movement scenes.

Pictured as free spaces with loose boundaries, the use of scenes in our research provide us with the flexibility to explore their conceptual boundaries with regards to food, health and labour. Moreover, it facilitates our understanding of the mechanisms at play and helps us to investigate the cultural characteristics linked both with the infiltration of SMOs in the unknown territories outside of street politics as well as to see how these scenes merge or contradict. At the same time, scenes are strongly bound to actual spaces (Haunss and Leach, 2007; Leach and Haunss, 2009), something which enables us to delve into more structural characteristics. Together with lifestyles and alternative cinema and music scenes, the empirical study of Hauns and Leach incorporates collectives, bookstores, and people's kitchens offering cheap meals, as important parts of the Autonomen social movement scene (Leach and Haunss, 2009). These examples do not only reflect the broader relevance with the selection of the cases under study, but also depict the particular features and characteristics the scenes demonstrate.

As bearers of movements' beliefs, values and history, social movement scenes play a significant role in maintaining the spark of social movement culture in times when mobilization is in decline. In this respect, Haunss and Leach argue that social movement scenes provide 'shelter for activists in times of low mobilization' (2007, p. 81), without this undermining their role in periods when mobilization is on the rise. Most importantly though, social movement scenes act as ways for people to engage with social movements. As we see also in the social movement scenes under study, and particularly in the social movement scene of health, participation in scenes requires less commitment and partial engagement. This, in turn, allows for the inclusion of non-activist audiences (Haunss and Leach, 2007). Although social movement scenes propose alternative organizational formats and prefigurative ideals, while bearing the cultural inheritance of social movements, the development of tension between social movements and scenes is not rare. As Haunss and Leach note, scenes tend to follow an experience-oriented path that contradicts the movements' attention on broader and long-term 
approaches (Ibid, p. 85). The authors' empirical research shows that these different orientations lead to conflicts, with the issue at stake being whether the movement should change its focus to enlarging its constituency outside of the scene's boundaries (Ibid, p. 84). These tensions mirror the dynamic condition of boundaries, where 'the meanings that activists and SMOs seek to establish through persuasion always have the potential to be transformed by supporters and sympathizers' (Steinberg, 2002, p. 213), is also reflected in the social movement scenes of our inquiry.

The aforementioned characteristics emphasize that scenes express cultural artifacts combined with material and structural dimensions. Moreover, they touch an audience which is not necessarily part of the social movement community but, nevertheless, shares with it a number of common features. These characteristics place the scenes in 'an intermediate location between the movement and broader political contexts', which have the potential to bridge 'the gap between cultural and structural approaches to movement dynamics' (Leach and Haunss, 2009, p. 276). In order to better expose and further boost these capabilities, we turn our attention to the factors of organizational structure, resources and identity.

\subsubsection{Organizational Structure, Resources and Identity}

In his literature review, Seferiades summarizes that the organization in social movements is typically approached as 1) institutionalized hierarchical structures; 2) the organization of collective action at the point where they meet with their opponents; and 3) cohesive structures and social networks that connect the leadership with the base (Seferiades, 2006). Probably accustomed to the third category, Elisabeth Clemens (Clemens, 1993) argues that organizational structure is a movement's first political action, as it pictures a way to publicize both the claims they make and the means they are going to use in order to achieve them. Both in empirical studies and in theoretical contributions, organizations, and particularly the factor of organizational structure of social movements and SMOs, have received a great deal of attention.

The importance of organizational structure usually preoccupies studies which concentrate on the differences between the old labour movement and new social movements. This difference is characterized by the distinct features of official and unofficial organizations. Contrary to official organizations, unofficial ones have a loose approach in terms of decision-making procedures, division of labour, criteria for membership and internal regulations (Gamson in Staggenborg, 2011, pp. 34-35). Despite some differences 
and adjustments (Della Porta, 2014, 2015; Della Porta and Mattoni, 2014), the SMOs which participated in the anti-austerity mobilizations employ these characteristics which were attributed earlier to unofficial organizations.

The difference between official and unofficial organizations became most obvious with the boom of organizational innovation in the 196os social movements (Tarrow, 1998, pp. 131-132). Among others, Diani notes that during periods of increased mobilization, new organizations and organizational models come to the forefront, while there is also an outbreak of agendas and tactics (Diani, 2015, p. 159). Together with the formation of new organizations, the literature of Contentious Politics claims that one key aspect of contentious cycles is the appropriation of old organizations. These concern the appropriation of political parties, unions and traditional SMOs by relatively new actors, responsible for affecting or changing the formers' trajectories. McAdam et al. (2001, p. 44) initially argued that 'instead of pointing to pre-existing mobilizing structures' as was the case in the USA, and the boom of 'organizational opportunities for collective action', we should turn our attention not to the creation of new organizations, but to how the existing ones are appropriated and transformed 'into vehicles of mobilization', providing this way a dynamic character for the stable formats of organizations. Almost ten years later, the authors asserted that their suggestion to move towards dynamic models had not yet been realized (McAdam et al., 2009). Although we do not claim to fill this gap, since appropriation in our case is not particularly related to the complete takeover of SMOs, our analysis adds some dynamic elements to the way scholars conceive organizations, by analyzing how the process of boundary enlargement affected the SMOs' repertoires and practices.

As Soule notes, organizations, and SMOs' organizational structure in particular, are quite important as they enforce the diffusion of tactics through the organizational collaboration (2013, p. 116). In this respect, organizations are closely related to boundaries, since organizational structure consists of an additional factor that groups use in order to enact boundaries that separate them from, or relate them with other groups (Reger, 2002, p. 173). Apart from revealing these characteristics with the use of the Contentious Politics approach, the factor of organizational structure helps us to understand how the process of boundary enlargement is shaped.

Along with the organizational structure, literature on social movements has paid great attention to resources. Scholars usually approach resources as factors which enable the sustenance of movements in time, but also as a means to achieve their goals (McCarthy and Zald, 1977). Resource mobilization theory has strongly contributed to the development of social movement 
studies by legitimizing collective action as a crucial way of doing politics (Edwards and McCarthy, 2004). Much attention to resources as a decisive factor for mobilization has, nevertheless, outweighed other important features, such as the external political environment or the activists' agency. Frame theorists were among the first to criticize the static role of resource mobilization theory, but they were not the only ones. As one would expect, the dynamic approach of Contentious Politics came into conflict with the resource mobilization theory, raising criticism against the structural and static approach of the latter (McAdam et al., 2001, pp. 44-50).

Although new theoretical frameworks have emerged and attention has moved away from resource mobilization theory, the same cannot be said for resources. Diani (2015) conceives resource allocation as one of the two deceiving factors (the other being boundary formation) responsible for distinguishing among the different modes of coordination. Taking these claims into consideration, boundary enlargement helps us to acquire a dynamic perspective on resources. Although we do not deny the role of resources as a crucial component needed by movements to attain their goals, we argue that in times of austerity, when wealth is scarce, resources acquire a substantial role and foster both activists' mobilization and the connection among SMOs. Local threats and constraints in movement resources favour the within-movement boundary-spanning and coalitions with taking place (Wang et al., 2018, pp. 172-173). This stands true also in our case, with the formation of coalitions within the three scenes, and particularly in the health scene, based on a great extent on the exchange of resources. In these terms, the factor of resources stands as a landmark in the process of boundary enlargement.

The factors of organizational structure and resources point towards the structural aspects of SMOs. However, in our attempt to explain the process of boundary enlargement, where previous fixed limits change shape and transform, it would be erroneous to omit the factor of identity. The cultural turn of social movement studies called for attention to the emotional and symbolic dimensions of movements. Conceiving movements as "discursive communities" is also linked with sharing common identities (Taylor, 2013, p. 43). These identities refer to "the shared sense of "one-ness" or "we-ness" anchored in real or imagined shared attributes' (Snow, 2013, pp. 266-267). Concerning the social movements' meso-level, identities indicate the 'cognitions shared by a group' (Taylor, 2013, p. 39). Snow argues that 'a relative pervasive or comprehensive identity is the one that that is thought of as thick identity, meaning the "student" in the classroom' (Snow, 2013, p. 269). However, since identities are subject to broader social transformations, 
they tend to be fluid and fragmented (Stekelenburg and Roggeband, 2013, pp. xvii-xviii).

Although Melucci's analyses acquire a dynamic perspective, the author finds it difficult to expose the processual approach to identity formation, since the term 'conveys too strongly the idea of the permanence of a subject' (Melucci, 1996, p. 85). Due to the lack of a more adequate term, the author underlines that the co-existence of different identities is because of the fact that none of them are self-sufficient; rather, each identity is mutually dependent on another (Melucci, 2002), something which emphasizes the complementing character of identities. This point, which also corresponds to the internal culture of scenes with the diverse identities of their participants, is central to the recognition of the symbiosis of the different identities; of which the connecting glue is solidarity (Melucci, 2002, p. 150; Snow, 2013, p. 267).

Polletta and Jasper define collective identity as 'an individual's cognitive, moral, and emotional connection with a broader community, category, practice, or institution' (2001, p. 85). As the authors suggest, this connection might be either real or imagined, and is separate from the personal identities (Ibid). Additional perspectives suggest that political culture should not be conceptualized as values and beliefs, but as frames which offer meanings to people with regards to specific situations. From this perceptive, according to Diani 'culture shapes how traits and relations combine in specific settings' (2015, p. 195). This is also reflected in Tarrow's account, which underlines that 'building a movement around strong ties of collective identity [...] does much of the work that would normally fall to organization; but it cannot do the work of mobilization, which depends on framing identities so that will lead to action, alliances, interaction' (Tarrow, 1998, p. 119). Among the processes that form collective identity in a group, such as the consciousness of common interests, the negotiation of its identity and the politicization of everyday life (Taylor and Whittier, 1992), boundaries are important in order to underline the differences between the members and non-members, dominants and subordinates (Taylor, 2013, p. 39). In this sense, collective identity constitutes a process under which the members of a group create the latter's cultural capital (Robnett, 2002, p. 267).

Following a post-structural approach, scholars underline that collective identities are 'dynamic, interactive, and socially constructed' (Taylor, 2013, p. 38). Coming from the structuralist background of the political process approach, the framework of Contentious Politics does not reject structures in favour of more cultural understandings; rather, it resonates that identities are inherent to structures. Without denying that external conditions and 
environmental constrains affect the formation of identities, we underline the role of individuals in shaping identities through their actions. Thus, identities should not be taken as given formations that obey traditions or as normative associations (Melucci, 1996, pp. 108-109). The creation of a collective identity is a long-term and difficult process. Collective identities are not static but change according to activists, environmental factors and the goals of the movements (Reger, 2002; Robnett, 2002, p. 268; Tarrow, 1998, p. 120). Among others, eventful protests (Della Porta, 2008; Della Porta et al., 2018, pp. 1-24) assist in the development of collective identities, which are also equipped with emotional booms, feelings of solidarity and moral shocks (Jasper and Poulsen, 1995).

Taking the aforementioned approach into consideration, Tilly and Tarrow (2006, p. 53) move a step beyond by arguing that political identities develop as a result of their interaction with political regimes. From this perspective, the exploration of political identities does not contradict the analysis of structures, but since the latter enforces or restricts the former, political identities are fundamental components of structures (Ibid, p. 69). The authors argue that collective identities are based on boundaries that distinguish "us from them", the relations with and across the boundaries, and employ common ways to conceive relations and boundaries (Tilly and Tarrow, 2015, p. 107). Taking into consideration the political opportunities and the movements' agency, the development of contentious identities stems from internal cognitive and external relational mechanisms (Tilly and Tarrow, 2006, p. 46). The next chapter points out some of the basic elements that characterize the social movement culture in Greece and how this has been constructed. Among a plethora of events, the recent economic crisis constitutes an additional factor that has affected this process, with the introduction of new heterogeneous actors and repertoires playing a crucial role.

Following a synthetic approach in which structural and cultural aspects complement each other, this exercise addresses two goals. First, by looking at factors of organizational structure and resources on the one hand, and at the factor of identity on the other, we further support the bridging of the often-conflicting theoretical approaches of resource mobilization theory and new social movement theories. The application of the Contentious Politics framework to the factors of organizational structure, resources and identity, enables us to conceptualize them as a continuum of SMOs' operation. At the same time, however, the exploration of the mechanisms that take place within each factor separately allows us to recognize the importance of these factors per se, which is the second goal. More precisely, this design gives us 
the opportunity to underline that organizational structure and resources, which have been underestimated by new social movement scholars, are still of great importance when it comes to the growth and development of SMOs. The same can be said for the factor of identity, which, contrary to structuralist conceptions, penetrates the fixed aspects of organization and resources and shapes the operations of SMOs. Overall, the application of the Contentious Politics approach to the organizational structure, resources and identity of the studied organizations allows them to be understood as a continuum, without losing their individual values.

\subsubsection{Sub-mechanisms, Combinations and Interconnections}

The mechanism-process approach of Contentious Politics is used to explain the how of collective action. Our attention to the three different social movement scenes lies in our effort to explain the different mechanisms that compose the process of boundary enlargement. Subsequently, by deconstructing each scene and analyzing it in terms of the factors of organizational structure, resources and identity, we are able to, on the one hand, demonstrate how the structural and cultural aspects blend together in the social movements' everyday context, and on the other hand, delve into the different trajectories that the mechanisms follow. However, researchers have been criticized for not revealing the "black boxes" of the studied mechanisms, meaning these elements that lie at the heart of each mechanism and make the latter's activation possible (Campbell, 2005). Alimi et al. (2015, p. 22) deal with this issue by arguing that the further deconstruction of mechanisms into sub-mechanisms can provide an answer, not only in terms of the activation of specific mechanisms, but also in order to explain dissimilarities among similar processes. Although we are a bit skeptical about whether this dismantling of mechanisms can be infinite, we adopt this suggestion and look deeper into how the mechanisms are being constructed.

The further exploration of the aforementioned mechanisms is not independent from the environment in which they operate. Research on social movements has recognized the three aspects of the organizational structure, resources, and identity probably as the most crucial points for the operation of SMOs. For analytical purposes, these aspects have been often distinguished by scholars in their effort to underline the weight of each one of them. Resource mobilization theory for example, emphasizes the role of organization and resources as the means that movements use to achieve their goals, while frame analysis raises questions regarding the construction of collective identity. However, these aspects are connected both practically 
and theoretically (Clemens, 1996). This leads us to suggest two things. First, as these three factors are often studied separately even though they are interlinked, the same can be said for the mechanisms that operate in the respective factors for each of our cases. In other words, although mechanisms are analyzed here separately, each mechanism percolates to the others and it is their combination that sets in motion the process of boundary enlargement. Second, each sub-mechanism that constitutes a mechanism tends to reflect the particular features of the factors it operates with (these being organizational structure, resources or identity). Taking into consideration that a process can be composed of different (sequences and combinations of) mechanisms as proposed by Alimi et al. (2015, pp. 28-30) modification to the original research strategy of McAdam et al. (2001), what we suggest here is that the identification of mechanisms and sub-mechanisms is strictly conditioned by the context in which they operate. Thus, the same mechanism that might take place in the clinics' organizational structure as well as in the cooperatives' resources, can be composed of sub-mechanisms which would be defined by the two respective factors. This does not aim to restrict the analytical usage of mechanisms. Rather, we try to bring to the forefront the importance of the three factors, particularly that of resources, which seems to acquire an advanced role in enlarging SMOs' boundaries during the period of severe austerity.

The Contentious Politics approach, as introduced by McAdam et al. (2001) as well as by the authors' later work (Tilly and Tarrow, 2015), contribute with two important suggestions which are highly inter-related. As the name of the framework suggests, the first deals with contention, meaning periods of increased, intense and often conflicting political activity. Although the framework of Contentious Politics has the potential to apply to different contexts, such as civil wars, revolutions and periods of democratization, empirical research tends to favour the study of increased political activity, perceiving social movements as their basic actors. Using earlier work of social movement scholars (Tarrow, 1998), Tilly and Tarrow propose a specific path in implementing the framework of Contentious Politics. In particular, the authors urge us to specify the site of contention and the conditions that surround it, then to identify the streams of contention as well as their outcomes, and finally, to deconstruct the streams into episodes of contention, which will allow researchers to understand the activation of different mechanisms as well as the process that these are composed by (Tilly and Tarrow, 2015, p. 242).

What stems from this brief methodological overview of Contentious Politics is the attention to contention. Contentious episodes are the cornerstone 
of the framework and the field which unravels the development of mechanisms. However, this does not fully reflect the adoption of a mechanismsprocess approach with regards to our inquiry. In particular, episodes of contention play quite an important role in our narrative. As the following chapter on the background context claims, the social movement community in Greece has experienced a number of crucial moments between 2008 and 2016, unique in their intensity and diverse in terms of their context. At the same time, however, the development of these alternative, service-oriented repertoires also takes place in periods of silence. As we describe further on, mobilization reached its peak in 2012 and then started to decline, while the SYRIZA, Synaspismos Rizospastikis Aristeras (Coalition of Radical Left)-led governmental coalition between 2015 and 2019 has negatively affected the outbreak of collective action. In other words, the interest of our research is not restricted to periods of increased protest activity; it also engages with the framework of Contentious Politics in periods of silence.

In line with its focus on episodes of contention, the second suggestion of the Contentious Politics framework deals with the sequence of the mechanisms. Specifically, each process under examination is compound to mechanisms that have been activated. Together with the development of contentious episodes, mechanisms are developed. This implies a linear logic, bounded in a chronological order, in which each mechanism sets in motion the activation of the next one. As the research of Alimi et al. (2015) shows, the same rationale also applies to the sub-mechanisms. However, our research does not completely adopt this perspective.

Each of the three scenes present different narratives that lead to distinct trajectories. Similar to the model of the Contentious Politics framework, the exploration of each trajectory allows us to emphasize the sequential development of mechanisms and sub-mechanisms. Nevertheless, this is not always the case. The variety of the actors involved in the social movement activities under study, as well as the unstable environment of austerity, set up obstacles to the development of linear accounts. This is to be expected, as the fragmentation of identities, and the post-modern view we described earlier, disturb the evolution of linear accounts. Depending on the scenes, some mechanisms are interrelated and develop interdependently, while others are always present but their tension grows due to specific contextual conditions. Additionally, some mechanisms take place simultaneously in irregular timeframes, while others are activated not because of the combination of some mechanisms, but by the mixture of specific features found in groups of mechanisms. In order to tackle these issues, when it is difficult to identify the chronological sequence for the evolution of mechanisms and 
sub-mechanisms, we try to develop a hierarchy based on the importance each mechanism carries, in terms of the overall process of boundary enlargement. In simpler terms, specific mechanisms and sub-mechanisms are found to hold different levels of importance within the set of mechanisms in the three social movement scenes. In this regard, the sets of mechanisms depend both on the chronological sequence and the particular effect of individual mechanisms and sub-mechanisms, and therefore have greater effects in the process of boundary enlargement.

By providing further information on the adjustment of the Contentious Politics framework regarding our inquiry, we recognize some important advantages that this brings to the exploration of the changes that took place in the social movement community in Greece. Nevertheless, the study of the movement community in the context of the Greek economic crisis does not only have benefits; it also comes with strict limitations. With this in mind, we move forward to present the basic theoretical and empirical limitations that this inquiry faces.

\subsection{Limitations}

Since this research focuses on contemporary events, this study does not have the advantage of taking a distant perspective. More precisely, mechanisms and processes are social phenomena which undergo changes through a given status quo. Therefore, the identification of these changes and their subsequent confirmation by the academic community, become more feasible when time has passed between the period which constitutes the object of the research and the period when the research is actually conducted. However, these considerations do not find a universal application, as the framework of Contentious Politics has been used by researchers who study current events. Papanikolopoulos (2016) inquiry regarding the December 2008 riots, as well as Tilly and Tarrow (2015, p. Ch. 10) attention to the anti-austerity mobilizations and the movement for marriage equality constitute some of the more pertinent examples.

The challenge of doing research on current phenomena does not seem to prevent us from using the framework of Contentious Politics. Nonetheless, we should still be quite cautious when it comes to the exploration of certain mechanisms and processes that are central to the creation, change or disappearance of social identities. As Melucci (2002) underlines, social identities in post-modern society have been formed through processes of transformation, where the previously essentialist approach changes into a dynamic one. Thus, moving ahead from a condition of assigned identities, individuals' identity 
is affected at multiple levels, both due to their own ability to do so, but also because of the great levels of uncertainty they experience. This change is based on a process of internal negotiation, in which the main priority is not the way that individuals differentiate themselves from the rest; rather, it is how individuals manage to achieve an internal unity on the subject amongst themselves. In Melucci's view, this is a long-lasting dynamic and relational procedure (Ibid: 21-28). Thus, it seems easier to speak in favour of the activation of a boundary by identifying specific elements that signal the creation of distinctive lines between two actors, or the certification of specific claims by an external authority, but it is more difficult to address the mechanism of social appropriation and the politicization of previously nonpolitical actors. This takes us on to our second limitation, which concerns the levels of analysis.

Following the framework of Contentious Politics as demonstrated in the Dynamics of Contention, our attention is drawn to the meso-level of collective action, which in the case of the anti-austerity mobilizations corresponds to the three respective SMIs and the organizations that operate within their boundaries. Subsequently, the identification of specific mechanisms and processes should also reflect the meso-level of analysis. This is actually the case, concerning how we proceed with the first two aspects of analysis, namely the organizational structure and the resources of the respective SMIs. Nonetheless, the aspect of identity becomes slightly more complicated, since we inevitably stress issues that also apply to the micro-level.

In particular, the mechanism of social appropriation signals a shift in the individuals' status quo and their role with regards to political activity. The same can be said of broader processes, for example mobilization, which lies at the core of contentious politics. However, the role of Contentious Politics should not be confused with earlier behavioural studies and theories of collective action which looked at individual motives that led to participation. Although looking at individuals' motives would add more insight to our empirical analysis, our research design is different in terms that it tries to demonstrate how the meso-level interacts with the micro. ${ }^{4}$ As many scholars have underlined (Kotronaki, 2015; Staggenborg, 2002), the meso-level reflects the life of a movement since it connects the macro socio-political processes with the micro-level of individual motives and interaction. Therefore, in the

4 The approach of Contentious Politics interacts as well with the macro-level. This becomes quite obvious when one looks at the framework's attention on political opportunities. Since we consider the interaction of the meso-level with the macro one quite obvious in all the studies that adopt this framework, we chose not to elaborate further. 
example of social clinics and the mechanism of social appropriation, we do not opt to demonstrate a comprehensive account of how individuals became politicized; rather, we try to explain how the respective SMIs enabled their politicization in relation to the macroeconomic, socioeconomic and political changes. For this reason, we approach the identity-related mechanisms and sub-mechanisms mostly from the side of the organizations and the way these have changed the individuals, and to a lesser extent, from the way that the individuals' identities have been shaped by their interaction with the organizations.

Our last limitation rests on the methodological approach of Contentious Politics, and specifically the identification of contentious episodes and events. Episodes and events of contention are used as points, either in order to trace the evolution of mechanisms and processes from one to another or as a particular event which needs further exploration. This approach has been stressed by many contentious politics scholars, with the initial introduction of the framework by McAdam et al. (2001) in Dynamics of Contention and its last advancement by Tilly and Tarrow's Contentious Politics (Tilly and Tarrow, 2015) being among the most popular ones. In the following chapter, where we describe the context of our study, we briefly mention some mechanisms and processes that took place in the evolution of the Greek social movement community. The diffusion of practices and procedures during the Global Justice Movement (GJM), the self-reflection process that followed the December 2008 riots and the decentralization process in the aftermath of the square movement are in line with the suggested methodology, since they trace specific processes during specific contentious events. However, this is not the case with the process of boundary enlargement and the shift of SMOs' attention towards service-oriented repertoires we try to demonstrate in this work.

Usually, the academic community situates the period of anti-austerity mobilizations between 2010 and 2012. The decline of protest repertoires and the rise of alternative repertoires is identified from 2012 onwards. ${ }^{5}$ However, we point out that the December 2008 riots played a crucial role in shaping the process of boundary enlargement. Therefore, within a broader time span, this process refers to the period between 2008 and 2016, without suggesting that these are either starting or end points respectively. During this period, a number of contentious events took place, something that we have taken into consideration. Nevertheless, the strict attachment of our design to these contentious events, as the Contentious Politics approach would have implied,

5 The increase here refers to the popularity of the alternative repertoire and not to the birth of its practices, since the latter finds mostly its starting point before the period of austerity. 
carries the risk of losing sight of the development of important mechanisms that do not comply with the appearance of these events.

A different approach used to trace important events would lead us to points where there were pronounced shifts in terms of service-related policies. Although the 4368/2016 law introduced by the SYRIZA-led government in August 2016 that gave the unemployed access to the public health system might signal a turning point for the operation of the social clinics, equally important developments, such as the rise of unemployment, could not be attributed to specific points in time, and therefore, prevent us from drawing a precise time period for the study of the clinics. The case becomes much more complicated when it comes to the social movement scenes of food and labour, where the implementation of specific policies seems to have little (if any) correlation to the rise in respective services.

These methodological difficulties complicate the explanation of the boundary enlargement process; however, they are not indestructible. As we have indicated earlier and we demonstrate in detail in our empirical chapters, the process of boundary enlargement is a gradual process, of which the vast development can be found within the period of 2008-2016. Therefore, although we underline the importance of some events and episodes of contention when possible, the overall explanation of this process does not necessitate their existence. Rather, the process of boundary enlargement is conceived as an on-going task which takes place within the studied period.

\subsection{Conclusion}

This chapter introduces the theoretical framework of the book which stands as our guide for the overall analysis of the effects of austerity on SMOs. Our argument is that the period of austerity acted as a catalyst that brought the process of boundary enlargement to the forefront. This process indicates that between 2008 and 2016, the fixed structural and cognitive boundaries of SMOs became more adaptable and ductile. This resulted in the incorporation of new and the modification of old elements within their operation. We do not support that this process was born during the period of crisis. Our approach understands social movement activity as a story of continuous struggles, interactions and conflicts which shapes its future practices. What we do argue, nevertheless, is that the needs created by austerity policies and politics have accelerated the enlargement of SMOs' boundaries.

One of the most interesting cases reflecting the process of boundary enlargement is the shift of SMOs' activity from a protest repertoire of action 
to one that is service-oriented. Among the large variety of services employed by SMOs, we focus on the social movement scenes of food, health and labour and the respective SMIs. In our effort to unravel the dynamic character of the boundary enlargement process and to deconstruct the mechanisms and sub-mechanisms that supported its activation, the framework of Contentious Politics is an adequate tool (McAdam et al., 2001). Although this framework proposes the identification of specific mechanisms and the subsequent composition of said mechanisms into a process, we follow the design of Alimi et al. (2015) and thus reverse the procedure by trying to understand what the combinations of mechanisms and sub-mechanisms that compose the proposed process are. Although we do not opt to provide an exhaustive account of the mechanisms and sub-mechanisms that form the process of boundary enlargement, we illustrate the most important ones in each of the studied social movement scenes. In these regards, the deconstruction of the respective SMIs regarding the organizational structure, resources and identity, enable the more precise description of the mechanisms and submechanisms. Apart from its direct utility, this deconstruction also achieves an indirect goal. That is to show that the blending of the two contradictory worlds, namely the structural world of the organizational structure and resources and the post-structural world of the identity factor, reflects the contradictions between the materialistic views and needs that emerged as a result of the austerity, and the post-materialistic approaches and values that the SMOs advocate for. It also stands as an indirect critique of the attribution of post-material views to the social movements positioned in the affluent societies of the developed North, contrary to the inferior materialistic ones of the less developed South. The process of mixing these two worlds is the theoretical value that the boundary enlargement process tries to bring to the forefront. Before we proceed with the analysis of our data, the next chapter aims to provide information regarding the background context of this study.

\section{Bibliography}

Ahrne, G., \& Brunsson, N. (2011). Organization Outside Organizations: The Significance of Partial Organization. Organization, 18(1), 83-104.

Alimi, E. Y., Bosi, L., \& Demetriou, C. (2015). The Dynamics of Radicalization: A Relational and Comparative Perspective. New York: Oxford University Press. Bauman, Z. (2007). Liquid Times: Living in an Age of Uncertainty. Cambridge, UK: Polity. 
Berman, M. (1983). All That Is Solid Melts into Air: The Experience of Modernity. London: Verso.

Bosi, L. (2016). Incorporation and Democratisation: The Long Term Process of Institutionalisation of the Northern Ireland Civil Rights Movement. In L. Bosi, M. Giugni, \& K. Uba (Eds.), The Consequences of Social Movements (pp. 338-360). Cambridge: Cambridge University Press,.

Campbell, J. L. (2005). Where Do We Stand? Common Mechanisms in Organizations and Social Movement Research. In D. McAdam, G. F. Davis, M. N. Zald, \& W. R. Scott (Eds.), Social Movements and Organization Theory (pp. 41-68). Cambridge: Cambridge University Press.

Clemens, E. S. (1993). Organizational Repertoires and Institutional Change: Women's Groups and the Transformation of Us Politics, 1890-1920. American journal of sociology, 98(4), 755-798.

Clemens, E. S. (1996). Organizational Form as Frame: Collective Identity and Political Strategy in the American Labor Movement, 1880-1920. In D. McAdam, J. D. McCarthy, \& M. N. Zald (Eds.), Comparative Perspectives on Social Movements: Political Opportunities, Mobilizing Structures, and Cultural Framings (pp. 205226). Cambridge Cambridge University Press.

Clemens, E. S. (2005). Two Kinds of Stuff: The Current Encounter of Social Movements and Organizations. In D. McAdam, G. F. Davis, M. N. Zald, \& W. R. Scott (Eds.), Social Movements and Organization Theory (pp. 351-366). Cambridge: Cambridge University Press.

Davis, G. F., McAdam, D., Scott, W. R., \& Zald, M. N. (2005). Social Movements and Organization Theory. New York: Cambridge University Press.

Dee, E. (2018). Institutionalization as Path to Autonomy: An Anarchist Social Center in Brighton. Space and Culture, 21(2), 192-204.

Della Porta, D. (2008). Eventful Protest, Global Conflicts. Distinktion: Scandinavian Journal of Social Theory, 9(2), 27-56.

Della Porta, D. (2014). Learning Democracy: Cross-Time Adaptation in Organisational Repertoires. In D. Della Porta \& A. Mattoni (Eds.), Spreading Protest: Movements in Times of Crisis (pp. 43-69). Colchester: ECPR Press.

Della Porta, D. (2015). Social Movements in Times of Austerity: Bringing Capitalism Back into Protest Analysis. Cambridge: Polity.

Della Porta, D., Andretta, M., Fernandes, T., Romanos, E., \& Vogiatzoglou, M. (2018). Legacies and Memories in Movements:Justice and Democracy in Southern Europe. New York: Oxford University Press.

Della Porta, D., \& Diani, M. (2006). Social Movements: An Introduction (2nd ed.). Malden, Mass.; Oxford: Blackwell.

Della Porta, D., \& Mattoni, A. (2014). Patterns of Diffusion and the Transnational Dimension of Protest in the Movements of the Crisis: An Introduction. In D. 
Della Porta \& A. Mattoni (Eds.), Spreading Protest. Social Movements in Times of Crisis (pp. 1-18). Colchester: ECPR Press.

Diani, M. (1992). The Concept of Social Movement. The Sociological Review, 40(1), 1-25. doi:10.1111/j.1467-954X.1992.tbo2943.x

Diani, M. (2013). Organizational Fields and Social Movement Dynamics. In The Future of Social Movement Research. Minneapolis: University of Minnesota Press. Diani, M. (2015). The Cement of Civil Society. Cambridge: Cambridge University Press. Diani, M., \& Mische, A. (2015). Network Approaches and Social Movements. In D. Dela Porta \& M. Diani (Eds.), The Oxford Handbook of Social Movements (pp. 306-325). New York: Oxford.

Fligstein, N., \& McAdam, D. (2012). A Theory of Fields. New York: Oxford University Press.

Haunss, S., \& Leach, D. K. (2007). Social Movement Scenes: Infrastructures of Opposition in Civil Society. In D. Purdue (Ed.), Civil Societies and Social Movements (pp. 85-101). Oxon: Routledge/ECPR Studies in European Political Science.

Jasper, J., \& Poulsen, J. (1995). Recruiting Strangers and Friends: Moral Shocks and Social Networks in Animal Rights and Anti-Nuclear Protests. Social Problems, 42(4), 493-512. doi:10.2307/3097043

Jasper, J. M., \& Duyvendak, J. W. (2015). Players and Arenas: The Interactive Dynamics of Protest. Amsterdam: Amsterdam University Press.

Klandermans, B., \& Oegema, D. (1987). Potentials, Networks, Motivations, and Barriers: Steps Towards Participation in Social Movements. American Sociological Review, 519-531.

Kotronaki, L. (2015). The Theory of Contentious Politics in the Practice of Social Movements: The Movement against the Neoliberal Globalization (Greece, 200o-20o6)

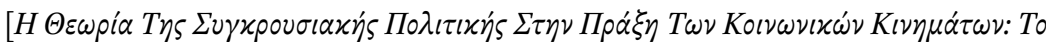

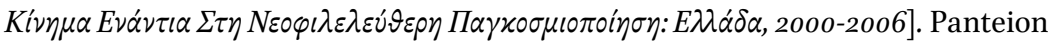
University of Social and Political Sciences. Retrieved from: https://www.didaktorika.gr/eadd/handle/10442/37556 Last Accessed: 28 April 2020

Kriesi, H. (1996). The Organizational Structure of New Social Movements in a Political Context. In D. McAdam, J. D. McCarthy, \& M. N. Zald (Eds.), Comparative Perspectives on Social Movements: Political Opportunities, Mobilizing Structures, and Cultural Framings (pp. 152-184). Cambridge: Cambridge University Press.

Leach, D. K., \& Haunss, S. (2009). Scenes and Social Movements. In H. Johnston (Ed.), Culture, Social Movements, and Protest. Farnham: Ashgate Publishing,Ltd. McAdam, D., Tarrow, S. G., \& Tilly, C. (2001). Dynamics of Contention. Cambridge, U.K.; New York: Cambridge University Press.

McAdam, D., Tarrow, S., \& Tilly, C. (2009). Comparative Perspectives on Contentious Politics. In M. I. Lichbach \& A. S. Zuckerman (Eds.), Comparative Politics: Rationality, Culture, and Structure (pp. 26o-29o). New York: Cambridge University Press. 
McCarthy, J. D., \& Zald, M. N. (1977). Resource Mobilization and Social Movements: A Partial Theory. American Journal of Sociology, 82(6), 1212-1241. doi:10.1086/226464 Melucci, A. (1996). Challenging Codes: Collective Action in the Information Age. Cambridge England; New York: Cambridge University Press.

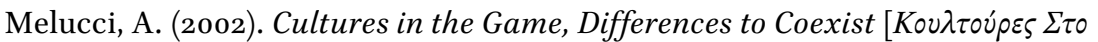

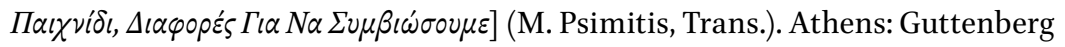
Papanikolopoulos, D. (2016). December 2008-Analysis and Interpretation: The Causal

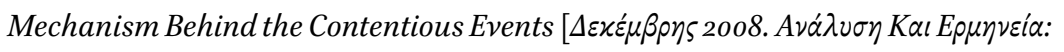

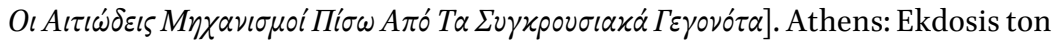
Sinadelfon

Polletta, F., \& Jasper, J. M. (2001). Collective Identity and Social Movements. Annual Review of Sociology, 27(1), 283-305.

Reger, J. (2002). More Than One Feminism: Organizational Structure and the Construction of Collective Identity. In D. S. Meyer, N. Whittier, \& B. Robnett (Eds.), Social Movements: Identity, Culture, and the State (pp. 171-184). New York: Oxford. Robnett, B. (2002). External Political Change, Collective Identities, and Participation in Social Movement Organizations. In Social Movements: Identity, Culture, and the State (pp. 266-285). New York: Oxford.

Rucht, D. (2013). Social Movement Structures in Action: Conceptual Propositions and Empirical Illustration. In J. v. Stekelenburg, C. Roggeband, \& B. Klandermans (Eds.), The Future of Social Movement Research:Dynamics, Mechanisms, and Processes (pp. 169-19o). Minneapolis: University of Minnesota Press.

Seferiades, S. (2006). Contentious Politics, Collective Action, Social Movements:

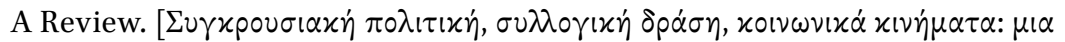
$\alpha \pi \circ \tau \dot{\pi} \pi \omega \sigma \eta]$. Greek Political Science Review, $27(1), 7-42$.

Snow, D. (2013). Identity Dilemmas, Discursive Fields, Identity Work, and Mobilization: Clarifying the Identity-Movement Nexus. In J. v. Stekelenburg, C. Roggeband, \& B. Klandermans (Eds.), The Future of Social Movement Research: Dynamics, Mechanisms, and Processes (pp. 263-280). Minneapolis: University of Minnesota Press.

Snow, D. A., Rochford Jr, E. B., Worden, S. K., \& Benford, R. D. (1986). Frame Alignment Processes, Micromobilization, and Movement Participation. American Sociological Review, 464-481.

Soule, S. A. (2013). Bringing Organizational Studies Back into Social Movement Scholarship. In J. v. Stekelenburg, C. Roggeband, \& B. Klandermans (Eds.), The Future of Social Movement Research: Dynamics, Mechanisms, and Processes (pp. 107-124). Minneapolis: Minnesota.

Staggenborg, S. (2002). The 'Meso' in Social Movement Research. In D. S. Meyer, N. Whittier, \& B. Robnett (Eds.), Social Movements: Identity, Culture, and the State (pp. 124-139). New York: Oxford.

Staggenborg, S. (2011). Social Movements (Rev. ed.). New York: Oxford University Press. 
Staggenborg, S. (2013). Organization and Community in Social Movements. In J. v. Stekelenburg, C. Roggeband, \& B. Klandermans (Eds.), The Future of Social Movement Research: Dynamics, Mechanisms, and Processes (pp. 125-144). Minneapolis: University of Minnesota Press.

Steinberg, M. W. (2002). Toward a More Dialogic Analysis of Social Movement Culture. In D. S. Meyer, N. Whittier, \& B. Robnett (Eds.), Social Movements: Identity, Culture, and the State (pp. 208-225). New York: Oxford.

Stekelenburg, J. v., \& Roggeband, C. (2013). Introduction: The Future of Social Movement Reserach. In J. v. Stekelenburg, C. Roggeband, \& B. Klandermans (Eds.), The Future of Social Movement Research: Dynamics, Mechanisms, and Processes. Minneapolis: Minnesota.

Stekelenburg, J. v., Roggeband, C., \& Klandermans, B. (2013). The Future of Social Movement Research Dynamics, Mechanisms, and ProcessesSocial movements, protest, and contention $v 39$ (pp. xxii, 469 p.).

Tarrow, S. (1998). Power in Movement: Social Movements and Contentious Politics (2nd ed ed.). Cambridge: Cambridge University Press.

Taylor, V. (2013). Social Movement Participation in the Global Society: Identity, Networks and Emotions. In J. v. Stekelenburg, C. Roggeband, \& B. Klandermans (Eds.), The Future of Social Movement Research:Dynamics, Mechanisms, and Processes (pp. 37-57). Minneapolis: University of Minnesota Press.

Tilly, C. (2004). Social Movements, 1768-2004. Boulder, Col: Paradigm Publishers.

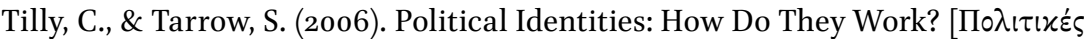

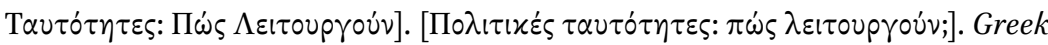
Political Science Review, 27(1), 43-70. doi:http://dx.doi.org/10.12681/hpsa.14672

Tilly, C., \& Tarrow, S. G. (2015). Contentious Politics (Second revised edition. ed.). New York, NY: Oxford University Press.

True, J. L., Jones, B. D., \& Baungartber, F. R. (2007). Punctuated-Equilibrium Theory: Explaining Stabilitity and Change in Public Policy. In P. A. Sabatier (Ed.), Theories of Policies Process (pp. 155-188). Boulder, CO: Westview Press.

Vandermerwe, S., \& Rada, J. (1988). Servitization of Business: Adding Value by Adding Services. European management journal, 6(4), 314-324.

Walgrave, S. (2013). Changing Mobilization of Individual Activists? In J. Van Stekelenburg, C. Roggeband, \& B. Klandermans (Eds.), The Future of Social Movement Research: Dynamics, Mechanisms, and Processes (pp. 205-215). Minneapolis: University of Minnesota Press.

Wang, D., Piazza, A., \& Soule, S. A. (2018). Boundary-Spanning in Social Movements: Antecedents and Outcomes. Annual Review of Sociology, 44, 167-187. 
